\title{
B4 androgen ablation: attacking the prostate cancer stem cell
}

\author{
Max S. Wicha \\ University of Michigan Comprehensive Cancer Center, Ann Arbor, Michigan, USA.
}

\begin{abstract}
There is increasing evidence that prostate cancers in rodent models and in men contain a cellular subpopulation that displays stem cell properties. These prostate cancer stem cells (PCSCs) lack androgen receptor expression and are increased in castration-resistant disease. In this issue of the JCI, a study from Yoshioka et al. demonstrates that PCSCs are regulated by a pathway in which $\alpha 6 \beta 4$ integrin amplifies signaling through ErbB2 and c-Met receptors. Targeting this pathway provides a novel therapeutic strategy for hormone refractory prostate cancer.
\end{abstract}

The prostate gland is thought to contain two distinct stem cell populations, one located in the basal layer and the other located within the luminal compartment. In mouse models, there is evidence that either of these primitive stem cells is capable of giving rise to prostate cancers (1). Development of the normal prostate gland and prostate cancers is dependent on androgenic hormones, the actions of which are mediated by the androgen receptor (AR). In the mouse, although orchiectomy results in marked prostate involution, the gland is completely regenerated following androgen replacement, suggesting the existence of castrationresistant stem cells. Similarly, although androgen ablation remains a mainstay for the treatment of advanced prostate cancer, hormone resistance inevitably develops and, as a result, hormonerefractory metastatic prostate cancer remains incurable. There is increasing evidence that many cancers, including those of the prostate, are driven and maintained by a cellular subpopulation that displays stem cell properties. These properties include self-renewal, which drives tumorigenesis, as well as differentiation, which generates the tumor bulk. Furthermore, studies suggest that unlike AR-driven bulk tumor cell populations, prostate cancer stem cells (PCSCs) lack AR and do not express differentia-

Conflict of interest: Max S. Wicha has financial holdings and is a scientific advisor for OncoMed Pharmaceuticals; is a scientific advisor for VeriStem, Paganini, and MedImmune; and receives research support from Dompe Pharmaceuticals and MedImmune.

Citation for this article: J Clin Invest. 2013; 123(2):563-565. doi:10.1172/JCI67460. tion markers, such as PSA (2). PCSCs are increased in hormone-refractory prostate cancer in patients as well as in mouse models. In addition to their role in hormone resistance, evidence suggests that these PCSCs may also mediate metastasis to sites such as the bone (3). Given their potential clinical importance, an understanding of the pathways that drive hormone-resistant PCSCs is of considerable importance. In this issue of the JCI, Yoshioka et al. use human prostate cancer specimens and mouse transgenic models to demonstrate an important role for $\beta 4$ integrin as well as the coexpressed receptor tyrosine kinases ErbB2 and c-Met in the regulation of PCSCs (4).

\section{The ties that bind}

Integrins are heterodimeric cell surface with extracellular matrix components. $\beta 4$ Integrin exclusively heterodimerizes with the $\alpha 6$ chain, and the resulting $\alpha 6 \beta 4$ heterodimer selectively binds to laminin-5, an important component of basement membranes. The interaction of laminin-5 with $\alpha 6 \beta 4$ integrin may anchor basal prostate stem cells to the basement membrane, constituting a normal prostate stem cell "niche" (5). Interestingly, in both mouse models and human disease, Yoshioka et al. found increased expression of $\beta 4$ integrin in castration-resistant compared with hormone-naive prostate cancer. Increased $\beta 4$ integrin expression was associated with a concomitant increase in expression and activation of the receptor tyrosine kinases ErbB2 and c-Met. Furthermore, cells expressing $\beta 4$ integrin, ErbB2, and c-Met also disreceptors that mediate interaction of cells played characteristics of PCSCs, including expression of the known markers CD49f ( $\alpha 6$ integrin) and Sca1, and the ability to initiate tumors when implanted into the renal capsules of immunosuppressed mice. Furthermore, Yoshioka et al. demonstrated that in mouse prostate cancer models pharmacologic inhibition of ErbB2 with lapatinib and c-Met with crizotinib induced apoptosis and inhibited tumor growth to a significantly greater extent than either agent alone, indicating that by driving the action of these two pathways $\beta 4$ integrin plays a critical role in maintaining PCSCs.

Similar signal transduction pathways may be involved in regulation of cancer stem cells (CSCs) in other tumor types. For example, $\beta 4$ integrin has previously been shown to amplify ErbB2 signaling in breast cancer cells, in which complexes of $\beta 4$ integrin and ErbB2 activate STAT3 signaling through transphosphorylation events (6). In human breast cancers, ErbB2 has been shown to be an important regulator of CSC self-renewal in a pathway involving ErbB2 activation of Akt with subsequent activation of Wnt signaling mediated by inactivation of GSK3 $\beta$ and facilitation of $\beta$-catenin nuclear translocation (7). c-Met has been reported to play a role in regulation of CSCs in hepatocellular and colorectal cancers, in which it provides a costimulatory signal activating the Wnt pathway (8). In addition, c-Met has previously been shown to induce a stem cell-like phenotype in human prostate cells (9). Given the observation that both ErbB2 and c-Met may regulate CSCs through Wnt pathway activation, it will be interesting to determine whether similar mechanisms regulate PCSCs.

\section{Unanswered questions}

Although the studies of Yoshioka et al. represent an important advance by demonstrating a role for $\beta 4$ integrin/ErbB2/c-Met signaling in PCSCs, a number of questions still remain. Putative PCSC markers have been described, including the $\alpha 6 \beta 4$ and 

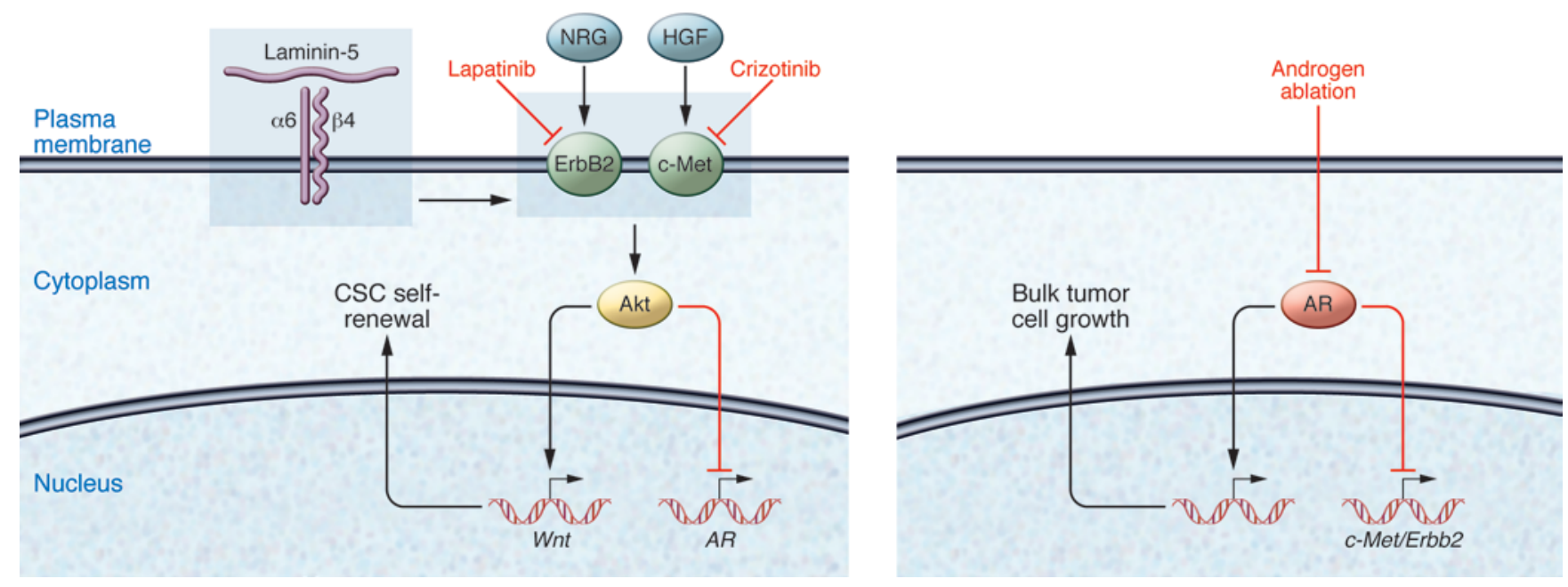

Figure 1

Regulation of CSC and bulk tumor cell population in prostate cancer. (A) CSCs are regulated by androgen-independent pathways, involving $\alpha 634$ integrin that amplifies signaling through ErbB2 and c-Met pathways, which can be inhibited by lapatinib and crizotinib. (B) Bulk tumor cell growth is primarily regulated by androgens through interaction with $\mathrm{AR}$ and is sensitive to androgen blockade. Androgen signaling inhibits expression of Erbb2 and $c-M e t$.

$\alpha 2 \beta 1$ integrins, aldehyde dehydrogenase, CD44, and CD133. It remains unclear, however, whether these markers identify a single PCSC population or multiple PCSC populations. Yoshioka et al. (4) used the currently accepted, state-of-the-art model for prostate tumor initiation, in which prostate cancer cells are implanted with urogenital mesenchyme under the renal capsule of immunosuppressed mice (10). However, Yoshioka et al., did not fractionate these cells to define the phenotype capable of tumor initiation in the model. It would be interesting to determine whether cells expressing putative PCSC markers had this capability.

It will also be important to ascertain which pathways downstream from ErbB2 and c-Met mediate cell survival and proliferation of PCSCs. As noted above, the possibility that these pathways converge to modulate Wnt signaling needs to be further explored. It will be important to ascertain the source of HGF and neuregulin, ligands that activate c-Met and ErbB signaling, respectively. Differentiated prostate cancer cells or stromal cells in the tumor microenvironment may constitute important sources of these ligands, which in turn might regulate PCSCs through paracrine interactions. Also relevant is whether androgens modulate paracrine interactions, as is the case for estrogen and progesterone in breast cancer $(11,12)$.

\section{Bench to bedside}

The studies of Yoshioka et al. also have important clinical implications. Current treatment strategies for prostate cancer involve administration of androgen ablative therapy early in the disease course, a treatment that is maintained until hormone resistance develops. Recent studies suggest that prostate cancers that become refractory to traditional hormone manipulations may still respond to newly developed hormone blocking agents such as abiraterone (13). However, despite the clinical benefit afforded by these new hormonal agents, hormone resistance inevitably develops and subsequent therapeutic options remain limited. Accumulating evidence, including this current work from Yoshioka et al., suggests that an additional important mechanism mediating castration-resistant disease is expansion of a hormone-independent CSC population driven by $\alpha 6 \beta 4$ integrin, ErbB2, and c-Met signaling. Although clinical trials using trastuzumab to block ErbB2 (14) or an antibody against the c-Met ligand HGF (15) failed to demonstrate clinical efficacy in advanced prostate cancer, a recent clinical trial demonstrated significant activity of cabozantinib, a c-Met inhibitor, in men with advanced castrationresistant prostate cancer (16). Furthermore, the studies of Yoshioka et al. suggest potentially more effective clinical strategies. In the preclinical models that they examined, the authors found that combined blockade of ErbB2 and c-Met was required to induce cellular apoptosis and significantly inhibit tumor growth, while each agent used alone had more modest effects. Furthermore, since CSCs constitute only a small subpopulation of the tumor, CSC-targeting agents would not be expected to produce tumor regression in advanced disease. In contrast, if CSCs mediate tumor growth at sites of micrometastasis, the greatest efficacy of these CSCtargeting agents would be when they are deployed in the adjuvant setting. Indeed, up to $30 \%$ of men with apparently localized prostate cancer harbor micrometastasis, as determined by bone marrow biopsy at the time of diagnosis (17). A similar clinical scenario exists in early-stage ErbB2-positive breast cancer, in which administration of the ErbB2 blocker trastuzumab in the adjuvant setting reduces recurrence by over 50\% (18). This suggests a strategy for the treatment of early-stage prostate cancer, involving simultaneous targeting of the PCSC population at sites of micrometastasis with combined ErbB2 and c-Met blockade, while simultaneously targeting bulk disease with androgen ablative therapy (Figure 1). By eliminating or reducing the PCSC population, such an approach has the potential to significantly delay or ultimately eliminate the development of hormone refractory metastatic disease. 


\section{Acknowledgments}

Max S. Wicha is supported by NIH grants (CA66233, CA101860, and P30CA46592), Susan G. Komen for the Cure, Breast Cancer Research Foundation, and the Taubman Research Institute.

Address correspondence to: Max S. Wicha, University of Michigan Comprehensive Cancer Center, 1500 East Medical Center Drive, Room 6302, Ann Arbor, Michigan 48109, USA. Phone: 734.936.1831; Fax: 734.615.3947; E-mail: mwicha@umich.edu.

1. Choi N, Zhang B, Zhang L, Ittmann M, Xin L. Adult murine prostate basal and luminal cells are self-sustained lineages that can both serve as targets for prostate cancer initiation. Cancer Cell. 2012;21(2):253-265.

2. Qin J, et al. The PSA(-/lo) prostate cancer cell population harbors self-renewing long-term tumorpropagating cells that resist castration. Cell Stem Cell. 2012;10(5):556-569.

3. Colombel M, et al. Increased expression of putative cancer stem cell markers in primary prostate cancer is associated with progression of bone metastases. Prostate. 2012;72(7):713-720.

4. Yoshioka T, et al. $\beta 4$ Integrin signaling induces expansion of prostate tumor progenitors. J Clin Invest. 2013;123(2):682-699.

5. Lang SH, Anderson E, Fordham R, Collins AT. Modeling the prostate stem cell niche: an evaluation of stem cell survival and expansion in vitro. Stem Cells Dev. 2010;19(4):537-546.

6. Guo W, et al. Beta 4 integrin amplifies ErbB2 signaling to promote mammary tumorigenesis. Cell. 2006;126(3):489-502.

7. Korkaya H, et al. Regulation of mammary stem/ progenitor cells by PTEN/Akt/beta-catenin signaling. PLoS Biol. 2009;7(6):e1000121.

8. Vermeulen L, et al. Wnt activity defines colon cancer stem cells and is regulated by the microenvironment. Nat Cell Biol. 2010;12(5):468-476.

9. van Leenders GJ, et al. Activation of c-MET induces a stem-like phenotype in human prostate cancer. PLoS One. 2011;6(11):e26753.

10. Goldstein AS, Huang J, Guo C, Garraway IP, Witte ON. Identification of a cell of origin for human prostate cancer. Science. 2010;329(5991):568-571.

11. Fillmore CM, et al. Estrogen expands breast cancer stem-like cells through paracrine FGF/ Tbx3 signaling. Proc Natl Acad Sci U S A. 2010; 107(50):21737-21742.

12. Joshi PA, et al. Progesterone induces adult mammary stem cell expansion. Nature. 2010; 465(7299):803-807.

13. Fizazi K, et al. Abiraterone acetate for treatment of metastatic castration-resistant prostate cancer: final overall survival analysis of the COUAA-301 randomised, double-blind, placebocontrolled phase 3 study. Lancet Oncol. 2012; 13(10):983-992.

14. Ziada A, et al. The use of trastuzumab in the treatment of hormone refractory prostate cancer; phase II trial. Prostate. 2004;60(4):332-337.

15. Ryan CJ, et al. Targeted MET inhibition in castration-resistant prostate cancer: a randomized phase II study and biomarker analysis with rilotumumab plus mitoxantrone and prednisone. Clin Cancer Res. 2013;19(1):215-224.

16. Smith DC, et al. Cabozantinib in patients with advanced prostate cancer: results of a phase II randomized discontinuation trial [published online ahead of print November 19, 2012]. J Clin Oncol. doi:10.1200/JCO.2012.45.0494.

17. Bretton PR, Melamed MR, Fair WR, Cote RJ. Detection of occult micrometastases in the bone marrow of patients with prostate carcinoma. Prostate. 1994;25(2):108-114.

18. Slamon DJ, et al. Use of chemotherapy plus a monoclonal antibody against HER2 for metastatic breast cancer that overexpresses HER2. $N$ Engl J Med. 2001;344(11):783-792. 